\title{
Impulsive stochastic fractional differential equations driven by fractional Brownian motion
}

\author{
Mahmoud Abouagwa ${ }^{1,2^{*}} \mathbb{D}$, Feifei Cheng ${ }^{2}$ and $\mathrm{Ji} \mathrm{Li}^{2}$
}

\section{"Correspondence:}

mahmoud.aboagwa@cu.edu.eg

'Department of Mathematical

Statistics, Faculty of Graduate

Studies for Statistical Research, Cairo

University, Giza, Egypt

${ }^{2}$ School of Mathematics and

Statistics, Huazhong University of

Science and Technology, Wuhan,

P.R. China

\section{Springer}

\begin{abstract}
In this research, we study the existence and uniqueness results for a new class of stochastic fractional differential equations with impulses driven by a standard Brownian motion and an independent fractional Brownian motion with Hurst index $1 / 2<H<1$ under a non-Lipschitz condition with the Lipschitz one as a particular case. Our analysis depends on an approximation scheme of Carathéodory type. Some previous results are improved and extended.
\end{abstract}

MSC: $34 \mathrm{~A} 37 ; 60 \mathrm{H} 10 ; 60 \mathrm{G} 22$

Keywords: Impulsive stochastic differential equations; Existence and uniqueness; Fractional calculus; Fractional Brownian motion

\section{Introduction}

Traditionally, a dominant interest in practical applications is the existence of solutions to deterministic fractional differential equations and fractional stochastic differential equations (FSDEs) driven by Brownain motion due to their role for helping candidates explore the hidden properties of the dynamics of complex systems in viscoelasticity, diffusion, mechanics, electromagnetism, control, signal processing, and physics. For example, in [28], the authors applied the concept of Caputo's $H$-differentiability to solve the fuzzy fractional differential equation with uncertainty. Benchaabane and Sakthivel [8] used the fractional calculus, semigroup theory, and stochastic analysis techniques to obtain the unique mild solution for a class of nonlinear fractional Sobolev-type SDEs with non-Lipschitz coefficients in Hilbert spaces under a new set of sufficient conditions. For further work on FSDEs and fractional differential equations (FDEs), we refer to $[5,6,12,15,19,26,29,31,32]$ and references therein.

However, random perturbations with long-range dependence abundantly exist in a wide range of physical phenomena, such as hydrology, mathematical finance, medicine and communication networks [21,33]. Correspondingly, fractional Brownian motion (fBm) with the Hurst index $H \in(1 / 2,1)$ has been suggested as a replacement of the standard Brownian motion in studying fractional stochastic systems as follows. Under a new set of sufficient conditions, Mourad et al. [20] investigated the approximate controllability for

(c) The Author(s) 2020. This article is licensed under a Creative Commons Attribution 4.0 International License, which permits use sharing, adaptation, distribution and reproduction in any medium or format, as long as you give appropriate credit to the original author(s) and the source, provide a link to the Creative Commons licence, and indicate if changes were made. The images or other third party material in this article are included in the article's Creative Commons licence, unless indicated otherwise in a credit line to the material. If material is not included in the article's Creative Commons licence and your intended use is not permitted by statutory regulation or exceeds the permitted use, you will need to obtain permission directly from the copyright holder. To view a copy of this licence, visit http://creativecommons.org/licenses/by/4.0/. 
Sobolev-type stochastic fractional control systems with $\mathrm{fBm}$ by using semigroup theory, fractional calculus, stochastic analysis, and Banach's fixed point theorem. Pei and Xu [28] derived the unique solution for non-Lipschitz SDEs with fBm by using successive approximations. Moreover, for a massive body of published studies covering the existence and uniqueness of FSDEs driven by $\mathrm{fBm}$; see $[23,24,35]$ and references therein.

On the other hand, the past recent years have seen a rapid development of the theory of impulsive effects in many evolutionary processes such as telecommunications, finance, electronics, economics, and mechanics, in which states are often subject to abrupt and short changes in discrete moments of time and can be neglected throughout the whole duration of the intended process [22]. In light of recent developments in the theory of SDEs, it is becoming extremely difficult to ignore the existence of impulsive effects. Therefore several studies have documented the effect of impulses in studying the SDEs driven by Brownian motion [18] and fBm; see $[10,11,13,14]$ and references therein.

To the best of our knowledge, there is no work yet reported in the literature on impulsive fractional stochastic differential equations driven by $\mathrm{fBm}$. Therefore, motivated by this fact and in order to close this gap, in this paper, we initiate a research on one of such equations. The specific objective of this study is to prove the existence and uniqueness of solutions to the following impulsive stochastic fractional differential equations (ISFDEs) driven by a standard Brownian motion and an independent $\mathrm{fBm}$ of the form:

$$
\left\{\begin{aligned}
& d X(t)= b(t, X(t)) d t+\sigma_{1}(t, X(t)) d W(t)+g(t, X(t)) d W^{H}(t) \\
&+\sigma_{2}(t, X(t))(d t)^{\alpha}, \quad t \in[0, T], t \neq t_{j}, 0<\alpha<1, \\
& \triangle X\left(t_{j}\right)= X\left(t_{j}^{+}\right)-X\left(t_{j}^{-}\right)=I_{j}\left(X\left(t_{j}\right)\right), \quad j=1,2, \ldots, m, \\
& X(0)=X_{0} \in \mathbb{R}^{d}
\end{aligned}\right.
$$

where $T \geq 0$ is a fixed horizon, $W^{H}$ is an $m$-dimensional $\mathrm{fBm}$ with $1 / 2<H<1$ independent of an $m$-dimensional standard Gaussian process $W(t), t \in[0, T]$. In what follows, $(\Omega, \mathcal{F}, P)$ is a complete probability space with probability measure $P$ on $\Omega$, and the filtration $\left\{\mathcal{F}_{t}\right\}_{t \geq 0}$ refers to the $\sigma$-field generated by $\left\{W^{H}(s), W(s), s \in[0, t]\right\}$ and satisfying the usual conditions, that is, it is right continuous, and $\mathcal{F}_{0}$ contains all $P$-null sets. Assume that $b, \sigma_{2}:[0, T] \times \mathbb{R}^{d} \longrightarrow \mathbb{R}^{d}$ and $\sigma_{1}, g:[0, T] \times \mathbb{R}^{d} \longrightarrow \mathbb{R}^{d \times m}$ are appropriate measurable functions. Here $I_{j} \in C\left(\mathbb{R}^{d}, \mathbb{R}^{d}\right)(j=1,2, \ldots, m)$ are bounded functions with fixed times $t_{j}$ satisfying $0=t_{0}<t_{1}<t_{2}<\cdots<t_{m}<T$, and $X\left(t_{j}^{+}\right)$and $X\left(t_{j}^{-}\right)$represent the right and left limits of $X(t)$ at time $t_{j}$. Further, $\triangle X\left(t_{j}\right)=X\left(t_{j}^{+}\right)-X\left(t_{j}^{-}\right)$determines the jump in the state $X$ at time $t_{j}$, where $I_{j}$ is the jump size. $X_{0}$ is an $\mathcal{F}_{0}$-measurable random variable satisfying $\mathbb{E}\left|X_{0}\right|^{2}<\infty$.

The class of Eqs. (1) has attracted our attention because of their applications in complex dynamic processes in sciences and engineering and modeling many phenomena in ecological and epidemiological processes of population dynamic perturbed by unavoidable noises under multitime scales [27]. Moreover, Eqs. (1) can be used as a model of many evolutionary processes where the noises are correlated and can be modeled by $\mathrm{fBm}$.

To summarize, our contribution here is the first attempt to consider the existence and uniqueness of solutions to ISFDEs driven by $\mathrm{fBm}$. We obtained our results on Eqs. (1) by using Carathéodory approximation [2,3] under non-Lipschitz (Taniguchi [34]) condition with Lipschitz one as a particular case. Moreover, the results are still new even when the 
coefficients of (1) satisfy the Lipschitz condition and under the non-Lipschitz condition used in [4], which is a particular case of our conditions. Finally, the obtained results extend and improve some published results of $[1,4,28,36]$.

This paper is outlined as follows. In Sect. 2, we provide necessary notions and preliminaries on the pathwise integrals with respect to $\mathrm{fBm}$ and hypotheses needed throughout the paper. We give our main results on the existence and uniqueness theorem for ISFDEs driven by a standard Brownian motion and an independent $\mathrm{fBm}$ given by (1) followed by some remarks and corollaries in Sect. 3.

\section{Preliminaries}

In this section, we review some basic notions and notations on the backward stochastic integral with respect to $\mathrm{fBm}$, and for more details, we refer to $[9,16,25]$. The $\mathrm{fBm}$ with the Hurst index $H \in\left(\frac{1}{2}, 1\right)$ is a centered Wiener process $W^{H}=\left\{W^{H}(t)\right\}_{0 \leq t \leq T}$ with the covariance function

$$
R(r, s)=\frac{1}{2}\left(s^{2 H}+r^{2 H}-|r-s|^{2 H}\right) .
$$

Let $\psi:[0, \infty) \times[0, \infty) \longrightarrow[0, \infty)$ be given as

$$
\psi(r, s)=H(2 H-1)|r-s|^{2 H-2}, \quad r, s \in \mathbb{R}^{+},
$$

where $H \in\left(\frac{1}{2}, 1\right)$, and define the space of Borel-measurable functions $h:[0, \infty) \longrightarrow[0, \infty)$

$$
L_{\psi}^{2}\left(\mathbb{R}^{+}\right)=\left\{h:\|h\|_{\psi}^{2}=\int_{0}^{\infty} \int_{0}^{\infty} h(r) h(s) \psi(r, s) d s d r<\infty\right\},
$$

which is a separable Hilbert space under the inner product

$$
\left\langle h_{1}, h_{2}\right\rangle_{\psi}=\int_{0}^{\infty} \int_{0}^{\infty} h_{1}(r) h_{2}(s) \psi(r, s) d s d r, \quad h_{1}, h_{2} \in L_{\psi}^{2}\left(\mathbb{R}^{+}\right) .
$$

For any integer $n \geq 1$, denote by $\mathcal{S}$ the set of smooth cylindrical random variables of the form

$$
F=h\left(W^{H}\left(\phi_{1}\right), W^{H}\left(\phi_{2}\right), \ldots, W^{H}\left(\phi_{n}\right)\right),
$$

where $h \in \mathcal{C}_{b}^{\infty}\left(\mathbb{R}^{n}\right)$ (i.e., $h$ and its partial derivatives of all orders are bounded), $\phi_{i} \in \mathcal{H}$ $(i=1,2, \ldots, n), \mathcal{H}$ is a Hilbert space [7] defined as the completion of measurable functions $\phi$ such that $\|\phi\|_{\psi}^{2}<\infty$. Denote by $\mathbb{D}^{1, p}(\mathcal{H})(p>0)$ the Sobolev space of $\mathcal{H}$-valued random variables with subspace $\mathbb{D}^{1, p}(|\mathcal{H}|)$.

The Malliavin $\psi$-derivative of a smooth and cylindrical random variable $F \in \mathcal{S}$ is defined as the $\mathcal{H}$-valued random variable

$$
D_{t}^{\psi} F=\int_{\mathbb{R}^{+}} \psi(t, v) D_{v}^{H} F d v
$$

where

$$
D^{H} F=\sum_{i=1}^{n} \frac{\partial h}{\partial x_{i}}\left(W^{H}\left(\phi_{1}\right), W^{H}\left(\phi_{2}\right), \ldots, W^{H}\left(\phi_{n}\right)\right) \phi_{i} .
$$


Definition 2.1 ([30]) Let $\eta(t), t \in[0, T]$, be a stochastic process with integrable trajectories. The backward stochastic integral $\int_{0}^{T} \eta(u) d^{+} W^{H}(u)$ of $\eta(t)$ with respect to $W^{H}(t)$ is given as

$$
\lim _{\epsilon \rightarrow 0} \int_{0}^{T} \eta(u)\left[\frac{W^{H}(u-\epsilon)-W^{H}(u)}{\epsilon}\right] d u,
$$

provided that the limit exists in probability.

According to Remark 1 and Lemma 2 in [36], the following lemma comes:

Lemma 2.1 Let $W^{H}(t)$ be an $f B m$ with Hurst index $H>\frac{1}{2}$, and let a stochastic process $\eta(t) \in \mathcal{L}_{\psi}[0, T] \cap \mathbb{D}^{1,2}(|\mathcal{H}|)$. Then for every $T<\infty$,

$$
\mathbb{E}\left[\int_{0}^{T} \eta(u) d^{+} W^{H}(u)\right]^{2} \leq 2 H T^{2 H-1} \mathbb{E}\left[\int_{0}^{T}|\eta(u)|^{2} d u\right]+4 T \mathbb{E} \int_{0}^{T}\left[D_{u}^{\psi} \eta(u)\right]^{2} d u .
$$

The following definition defines the integration with respect to $(d t)^{\beta}$, and the reader is referred to [17] for the proof.

Definition 2.2 Let $g(t)$ be a continuous function. Then its integral with respect to $(d t)^{\beta}$, $0<\beta \leq 1$, is defined by

$$
\int_{0}^{t} g(s)(d s)^{\alpha} \beta=\beta \int_{0}^{t}(t-s)^{\beta-1} g(s) d s, \quad 0<\beta \leq 1 .
$$

Similar to Definition 2.2 in [1], the definition of the unique solution to Eq. (1) can be given as follows.

Definition 2.3 An $\mathbb{R}^{d}$-valued stochastic process $X(t), t \in[0, T]$, is called a unique solution to Eq. (1) if:

(i) $X(t)$ is $\mathcal{F}_{t}$-adapted;

(ii) For every $t \in[0, T], X(t)$ satisfies the following integral equation:

$$
\begin{aligned}
X(t)= & X_{0}+\int_{0}^{t} b(s, X(s)) d s+\int_{0}^{t} \sigma_{1}(s, X(s)) d W(s)+\int_{0}^{t} g(s, X(s)) d^{+} W^{H}(s) \\
& +\alpha \int_{0}^{t} \frac{\sigma_{2}(s, X(s))}{(t-s)^{1-\alpha}} d s+\sum_{0<t_{j}<t} I_{j}\left(X\left(t_{j}\right)\right) \quad \mathbb{P} \text {-a.s.; }
\end{aligned}
$$

(iii) For any other solution $Y(t)$, we have $P\{X(t)=Y(t), \forall 0 \leq t \leq T\}=1$.

To attain the main results, the following assumptions are imposed on the coefficients $b$, $\sigma_{1}, g$, and $\sigma_{2}$.

(H1) For all $t \in[0, T]$ and $b(t, \cdot), \sigma_{1}(t, \cdot), g(t, \cdot), \sigma_{2}(t, \cdot) \in \mathcal{L}_{\psi}[0, T] \cap \mathbb{D}^{1,2}(|\mathcal{H}|)$, we have

$$
|b(t, X)|^{2}+\left|\sigma_{1}(t, X)\right|^{2}+|g(t, X)|^{2}+\left|D_{t}^{\psi} g(t, X)\right|^{2}+\left|\sigma_{2}(t, X)\right|^{2} \leq \mathcal{R}\left(t,|X|^{2}\right),
$$

where $\mathcal{R}(t, v):[0,+\infty) \times \mathbb{R}^{+} \longrightarrow \mathbb{R}^{+}$is a function locally integrable in $t$ for any fixed $v \geq 0$ and continuous, nondecreasing, and concave in $v$ for any fixed 
$t \in[0, T]$. Further, the integral equation

$$
v(t)=v_{0}+K \int_{0}^{t} \mathcal{R}(s, v(s)) d s
$$

has a global solution on $[0, T]$ for all $K>0$ and $v_{0} \geq 0$.

(H2) For all $t \in[0, T]$ and $b(t, \cdot), \sigma_{1}(t, \cdot), g(t, \cdot), \sigma_{2}(t, \cdot) \in \mathcal{L}_{\psi}[0, T] \cap \mathbb{D}^{1,2}(|\mathcal{H}|)$, we have

$$
\begin{aligned}
& |b(t, X)-b(t, Y)|^{2}+\left|\sigma_{1}(t, X)-\sigma_{1}(t, Y)\right|^{2}+|g(t, X)-g(t, Y)|^{2} \\
& \quad+\left|D_{t}^{\psi}(g(t, X)-g(t, Y))\right|^{2}+\left|\sigma_{2}(t, X)-\sigma_{2}(t, Y)\right|^{2} \leq \mathcal{G}\left(t,|X-Y|^{2}\right),
\end{aligned}
$$

where $\mathcal{G}:[0,+\infty) \times \mathbb{R}^{+} \longrightarrow \mathbb{R}^{+}$is a function locally integrable in $t$ for any fixed $v \geq 0$ and continuous, nondecreasing, and concave in $v$ for any fixed $t \in[0, T]$ such that $\mathcal{G}(t, 0)=0$ and $\int_{0+} \frac{1}{\mathcal{G}(t, v)} d v=+\infty$. Moreover, for $\lambda>0$, every $t \in[0, T]$, and every nonnegative continuous function $\mathcal{M}(t)$ such that

$$
\left\{\begin{array}{l}
\mathcal{M}(t) \leq \lambda \int_{0}^{t} \mathcal{G}(s, \mathcal{M}(s)) d s, \quad t \in \mathbb{R} \\
\mathcal{M}(0)=0
\end{array}\right.
$$

we have $\mathcal{M}(t) \equiv 0$.

(H3) There exist some positive constants $d_{j}(j=1,2, \ldots)$ such that

$$
\left|I_{j}(X)-I_{j}(Y)\right| \leq d_{j}|X-Y|
$$

for all $X, Y \in \mathcal{L}_{\psi}[0, T] \cap \mathbb{D}^{1,2}(|\mathcal{H}|)$ and $\left|I_{j}(0)\right|=0$.

\section{Main results}

In this section, we present the existence and uniqueness of solutions to Eq. (1).

Theorem 3.1 Let hypotheses (H1)-(H3) be satisfied, and let $X_{0}$ be independent of the Brownian motion $W(s)$ and the $f B m W^{H}(s)(s>0, H>1 / 2)$ with finite second moment. Then there exists a unique solution $X(t)$ to $E q$. (1) on $[0, T]$, provided that $10 m \sum_{j=1}^{m}\left(d_{j}\right)^{2}<1$.

Proof To begin with, we introduce the Carathéodory approximation as follows. For any integer $n \geq 1$, define $X_{n}(t)=X(0)=X_{0}$ for all $-1 \leq t \leq 0$ and

$$
\begin{aligned}
X_{n}(t)= & X_{0}+\int_{0}^{t} b\left(s, X_{n}\left(s-\frac{1}{n}\right)\right) d s+\int_{0}^{t} \sigma_{1}\left(s, X_{n}\left(s-\frac{1}{n}\right)\right) d W(s) \\
& +\int_{0}^{t} g\left(s, X_{n}\left(s-\frac{1}{n}\right)\right) d^{+} W^{H}(s)+\alpha \int_{0}^{t} \frac{\sigma_{2}\left(s, X_{n}\left(s-\frac{1}{n}\right)\right)}{(t-s)^{1-\alpha}} d s \\
& +\sum_{0<t_{j}<t} I_{j}\left(X_{n}\left(t_{j}-\frac{1}{n}\right)\right), \quad 0 \leq t \leq T .
\end{aligned}
$$

We split the proof into the following three parts.

Part 1. For all $t \in[0, T]$, the sequence $\left\{X_{n}(t)\right\}_{n \geq 1}$ is bounded. 
By the Hölder and Burkholder-Davis-Gundy (B-D-G) inequalities and Lemma 2.1 from Eq. (3) we have

$$
\begin{aligned}
\mathbb{E}\left(\sup _{0 \leq s \leq t}\left|X_{n}(s)\right|^{2}\right) \leq & 6 \mathbb{E}\left|X_{0}\right|^{2}+6 T \mathbb{E} \int_{0}^{T}\left|b\left(s, X_{n}\left(s-\frac{1}{n}\right)\right)\right|^{2} d s \\
& +24 \mathbb{E} \int_{0}^{t}\left|\sigma_{1}\left(s, X_{n}\left(s-\frac{1}{n}\right)\right)\right|^{2} d s \\
& +12 H T^{2 H-1} \mathbb{E} \int_{0}^{t}\left|g\left(s, X_{n}\left(s-\frac{1}{n}\right)\right)\right|^{2} d s \\
& +24 T \mathbb{E} \int_{0}^{t}\left|D_{s}^{\psi} g\left(s, X_{n}\left(s-\frac{1}{n}\right)\right)\right|^{2} d s \\
& +6 \alpha^{2} \frac{T^{2 \alpha-1}}{2 \alpha-1} \mathbb{E} \int_{0}^{t}\left|\sigma_{2}\left(s, X_{n}\left(s-\frac{1}{n}\right)\right)\right|^{2} d s \\
& +6 m \mathbb{E} \sum_{j=1}^{m}\left|I_{j}\left(X_{n}\left(t_{j}-\frac{1}{n}\right)\right)\right|^{2}(\alpha \in(1 / 2,1)) .
\end{aligned}
$$

Thus by conditions (H1) and (H3) and the Jensen inequality we have

$$
\begin{aligned}
\mathbb{E}\left(\sup _{0 \leq s \leq t}\left|X_{n}(s)\right|^{2}\right) \leq & 6 \mathbb{E}\left|X_{0}\right|^{2}+6 C_{1} \mathbb{E} \int_{0}^{t} \mathcal{R}\left(s,\left|X_{n}\left(s-\frac{1}{n}\right)\right|^{2}\right) d s \\
& +6 m \sum_{j=1}^{m}\left(d_{j}\right)^{2} \mathbb{E}\left|X_{n}\left(t_{j}-\frac{1}{n}\right)\right|^{2} \\
\leq & 6 \mathbb{E}\left|X_{0}\right|^{2}+6 C_{1} \int_{0}^{t} \mathcal{R}\left(s, \mathbb{E}\left(\sup _{0 \leq u \leq s}\left|X_{n}(u)\right|^{2}\right)\right) d s \\
& +6 m \sum_{j=1}^{m}\left(d_{j}\right)^{2} \mathbb{E}\left(\sup _{0 \leq u \leq t}\left|X_{n}(u)\right|^{2}\right),
\end{aligned}
$$

which implies that

$$
\mathbb{E}\left(\sup _{0 \leq s \leq t}\left|X_{n}(s)\right|^{2}\right) \leq C_{2} \mathbb{E}\left|X_{0}\right|^{2}+C_{3} \int_{0}^{t} \mathcal{R}\left(s, \mathbb{E}\left(\sup _{0 \leq u \leq s}\left|X_{n}(u)\right|^{2}\right)\right) d s,
$$

where $C_{1}=\left[4+5 T+2 H T^{2 H-1}+\alpha^{2} \frac{T^{2 \alpha-1}}{2 \alpha-1}\right], C_{2}=\frac{6}{1-6 m \sum_{j=1}^{m}\left(d_{j}\right)^{2}}$, and $C_{3}=\frac{6 C_{1}}{1-6 m \sum_{j=1}^{m}\left(d_{j}\right)^{2}}$.

Now, by condition (H1) there exists a solution $u(t), t \in[0, T]$, satisfying

$$
u(t)=C_{2} \mathbb{E}\left|X_{0}\right|^{2}+C_{3} \int_{0}^{t} \mathcal{R}(s, u(s)) d s
$$

Comparing this above equation and Eq. (4), we have

$$
\mathbb{E}\left(\sup _{0 \leq s \leq t}\left|X_{n}(s)\right|^{2}\right) \leq u(t) \leq u(T)<\infty, \quad n \geq 1,
$$

which shows the uniform boundedness of $\left\{X_{n}(t)\right\}_{n \geq 1}$. 
Part 2. For $0 \leq s<t \leq T$ and integer $n \geq 1$, we claim that

$$
\mathbb{E}\left|X_{n}(t)-X_{n}(s)\right|^{2} \leq C_{4}(t-s)+C_{6}(t-s)^{2 \alpha}+C_{5} \sum_{s<t_{j}<t} C,
$$

where $C_{4}, C_{5}, C_{6}$ will be defined further in the proof, and the constant $C$ comes from Part 1.

Note that

$$
\begin{aligned}
& \left|X_{n}(t)-X_{n}(s)\right|^{2} \\
& \leq 5\left|\int_{s}^{t} b\left(u, X_{n}\left(u-\frac{1}{n}\right)\right) d u\right|^{2}+5\left|\int_{s}^{t} \sigma_{1}\left(u, X_{n}\left(u-\frac{1}{n}\right)\right) d W(u)\right|^{2} \\
& \quad+5\left|\int_{s}^{t} g\left(u, X_{n}\left(u-\frac{1}{n}\right)\right) d^{+} W^{H}(u)\right|^{2}+5\left|\sum_{s<t_{j}<t} I_{j}\left(X_{n}\left(t_{j}-\frac{1}{n}\right)\right)\right|^{2} \\
& \quad+5 \alpha^{2} \mid \int_{0}^{s}\left(\frac{\sigma_{2}\left(u, X_{n}\left(u-\frac{1}{n}\right)\right)}{(t-u)^{1-\alpha}}-\frac{\sigma_{2}\left(u, X_{n}\left(u-\frac{1}{n}\right)\right)}{\left.(s-u)^{1-\alpha}\right)}\right) d u \\
& \quad+\left.\int_{s}^{t} \frac{\sigma_{2}\left(u, X_{n}\left(u-\frac{1}{n}\right)\right)}{(t-u)^{1-\alpha}} d u\right|^{2} \\
& \quad:=\sum_{i=1}^{5} I_{i} .
\end{aligned}
$$

Taking the expectation and using Itô isometry, Lemma 2.1, and (H1), we get

$$
\begin{aligned}
\mathbb{E}\left|I_{1}\right|+\mathbb{E}\left|I_{2}\right|+\mathbb{E}\left|I_{3}\right| & \leq 5(T-s) \int_{s}^{t} \mathbb{E}\left|b\left(u, X_{n}\left(u-\frac{1}{n}\right)\right)\right|^{2} d u+5 \int_{s}^{t} \mathbb{E}\left|\sigma_{1}\left(u, X_{n}\left(u-\frac{1}{n}\right)\right)\right|^{2} d u \\
& +10 H(T-s)^{2 H-1} \int_{s}^{t} \mathbb{E}\left|g\left(u, X_{n}\left(u-\frac{1}{n}\right)\right)\right|^{2} d u \\
& +20(T-s) \int_{s}^{t} \mathbb{E}\left|D_{u}^{\psi} g\left(u, X_{n}\left(u-\frac{1}{n}\right)\right)\right|^{2} d u \\
\leq & 5\left[1+5(T-s)+2 H(T-s)^{2 H-1}\right] \int_{s}^{t} \mathcal{R}\left(u, \mathbb{E}\left|X_{n}\left(u-\frac{1}{n}\right)\right|^{2}\right) d u \\
\leq & 5\left[1+5(T-s)+2 H(T-s)^{2 H-1}\right] \int_{s}^{t} \mathcal{R}\left(u, \mathbb{E}\left(\sup _{0 \leq v \leq u}\left|X_{n}(v)\right|^{2}\right)\right) d u
\end{aligned}
$$

which, via Part 1, gives

$$
\mathbb{E}\left|I_{1}\right|+\mathbb{E}\left|I_{2}\right|+\mathbb{E}\left|I_{3}\right| \leq C_{4}(t-s),
$$

where $C_{4}=5\left[1+5(T-s)+2 H(T-s)^{2 H-1}\right]\left(\sup _{0 \leq t \leq T} \mathcal{R}(t, C)\right)>0$. 
Abouagwa et al. Advances in Difference Equations

(2020) 2020:57

Page 8 of 14

Now, using Hölder's and Young's inequalities and conditions (H1) and (H3) gives

$$
\begin{aligned}
\mathbb{E}\left|I_{4}\right| & \leq 5 \mathbb{E}\left(\sum_{s<t_{j}<t}\left|I_{j}\left(X_{n}\left(t_{j}-\frac{1}{n}\right)\right)\right|\right)^{2} \leq 5 \mathbb{E}\left(\sum_{s<t_{j}<t} d_{j}\left|X_{n}\left(t_{j}-\frac{1}{n}\right)\right|\right)^{2} \\
& \leq 5 \sum_{s<t_{j}<t}\left(d_{j}\right)^{2} \sum_{s<t_{j}<t} \mathbb{E}\left|X_{n}\left(t_{j}-\frac{1}{n}\right)\right|^{2} \\
& \leq 5 \sum_{s<t_{j}<t}\left(d_{j}\right)^{2} \sum_{s<t_{j}<t} \mathbb{E}\left(\sup _{0 \leq u \leq t}\left|X_{n}(u)\right|^{2}\right) \leq C_{5} \sum_{s<t_{j}<t} C
\end{aligned}
$$

and

$$
\begin{aligned}
\mathbb{E}\left|I_{5}\right|= & 5 \alpha^{2} \mathbb{E} \mid \int_{0}^{s}\left((t-u)^{\alpha-1}-(s-u)^{\alpha-1}\right) \sigma_{2}\left(u, X_{n}\left(u-\frac{1}{n}\right)\right) d u \\
& +\left.\int_{s}^{t}(t-u)^{\alpha-1} \sigma_{2}\left(u, X_{n}\left(u-\frac{1}{n}\right)\right) d u\right|^{2} \\
\leq & 10 \alpha^{2} \int_{0}^{s}\left((t-u)^{\alpha-1}-(s-u)^{\alpha-1}\right) \mathbb{E}\left|\sigma_{2}\left(u, X_{n}\left(u-\frac{1}{n}\right)\right)\right|^{2} d u \\
& \times \int_{0}^{s}\left((t-u)^{\alpha-1}-(s-u)^{\alpha-1}\right) d u+10 \alpha^{2} \int_{s}^{t}(t-u)^{\alpha-1} d u \\
& \times \int_{s}^{t}(t-u)^{\alpha-1} \mathbb{E}\left|\sigma_{2}\left(u, X_{n}\left(u-\frac{1}{n}\right)\right)\right|^{2} d u \\
\leq & 10\left(\sup _{0 \leq t \leq T} \mathcal{R}(t, C)\right)\left(t^{\alpha}-s^{\alpha}+(t-s)^{\alpha}\right)^{2}+10\left(\sup _{0 \leq t \leq T} \mathcal{R}(t, C)\right)(t-s)^{2 \alpha} \\
\leq & C_{6}(t-s)^{2 \alpha},
\end{aligned}
$$

where $C_{5}=5 \sum_{s<t_{j}<t}\left(d_{j}\right)^{2}$ and $C_{6}=20\left(\sup _{0 \leq t \leq T} \mathcal{R}(t, C)\right)$ are positive constants.

Taking the expectation to Eq. (5), by Eqs. (6)-(8) we obtain the required result, and the proof of Part 2 is complete.

Part 3. The sequence $\left\{X_{n}(t)\right\}_{n \geq 1}$ is a Cauchy sequence. From Eq. (3), for $m>n \geq 1$ and $t \in[0, T]$, we easily get

$$
\begin{aligned}
& \mathbb{E}\left(\sup _{0 \leq s \leq t}\left|X_{m}(s)-X_{n}(s)\right|^{2}\right) \\
& \leq 5 \mathbb{E}\left(\sup _{0 \leq s \leq t}\left|\int_{0}^{s}\left[b\left(u, X_{m}\left(u-\frac{1}{m}\right)\right)-b\left(u, X_{n}\left(u-\frac{1}{n}\right)\right)\right] d u\right|^{2}\right) \\
& \quad+5 \mathbb{E}\left(\sup _{0 \leq s \leq t}\left|\int_{0}^{s}\left[\sigma_{1}\left(u, X_{m}\left(u-\frac{1}{m}\right)\right)-\sigma_{1}\left(u, X_{n}\left(u-\frac{1}{n}\right)\right)\right] d W(u)\right|^{2}\right) \\
& +5 \mathbb{E}\left(\sup _{0 \leq s \leq t}\left|\int_{0}^{s}\left[g\left(u, X_{m}\left(u-\frac{1}{m}\right)\right)-g\left(u, X_{n}\left(u-\frac{1}{n}\right)\right)\right] d^{+} W^{H}(u)\right|^{2}\right) \\
& \quad+5 \alpha^{2} \mathbb{E}\left(\sup _{0 \leq s \leq t}\left|\int_{0}^{s} \frac{\left[\sigma_{2}\left(u, X_{m}\left(u-\frac{1}{m}\right)\right)-\sigma_{2}\left(u, X_{n}\left(u-\frac{1}{n}\right)\right)\right]}{(s-u)^{1-\alpha}} d u\right|^{2}\right)
\end{aligned}
$$




$$
\begin{aligned}
& \quad+5 \mathbb{E}\left(\sup _{0 \leq s \leq t}\left|\sum_{0<u_{j}<s}\left[I_{j}\left(X_{m}\left(u_{j}-\frac{1}{m}\right)\right)-I_{j}\left(X_{n}\left(u_{j}-\frac{1}{n}\right)\right)\right]\right|^{2}\right) \\
& :=\sum_{i=1}^{5} J_{i} .
\end{aligned}
$$

By the plus and minus technique and assumption (H2) this yields

$$
\begin{aligned}
J_{1}+J_{4} \leq & 5 T \mathbb{E} \int_{0}^{t}\left|b\left(u, X_{m}\left(u-\frac{1}{m}\right)\right)-b\left(u, X_{n}\left(u-\frac{1}{n}\right)\right)\right|^{2} d u \\
& +5 \alpha^{2} \frac{T^{2 \alpha-1}}{2 \alpha-1} \mathbb{E} \int_{0}^{t}\left|\sigma_{2}\left(u, X_{m}\left(u-\frac{1}{m}\right)\right)-\sigma_{2}\left(u, X_{n}\left(u-\frac{1}{n}\right)\right)\right|^{2} d u \\
\leq & 10 T \mathbb{E} \int_{0}^{t}\left|b\left(u, X_{m}\left(u-\frac{1}{m}\right)\right)-b\left(u, X_{n}\left(u-\frac{1}{m}\right)\right)\right|^{2} d u \\
& +10 T \mathbb{E} \int_{0}^{t}\left|b\left(u, X_{n}\left(u-\frac{1}{m}\right)\right)-b\left(u, X_{n}\left(u-\frac{1}{n}\right)\right)\right|^{2} d u \\
& +10 \alpha^{2} \frac{T^{2 \alpha-1}}{2 \alpha-1} \mathbb{E} \int_{0}^{t} \mid \sigma_{2}\left(u, X_{m}\left(u-\frac{1}{m}\right)\right)-\sigma_{2}\left(u,\left.X_{n}\left(u-\frac{1}{m}\right)\right|^{2} d u\right. \\
& +10 \alpha^{2} \frac{T^{2 \alpha-1}}{2 \alpha-1} \int_{0}^{t}\left|\sigma_{2}\left(u, X_{n}\left(u-\frac{1}{m}\right)\right)-\sigma_{2}\left(u, X_{n}\left(u-\frac{1}{n}\right)\right)\right|^{2} d u \\
\leq & 10\left[T+\alpha^{2} \frac{T^{2 \alpha-1}}{2 \alpha-1}\right] \int_{0}^{t} \mathcal{G}\left(u, \mathbb{E}\left|X_{m}\left(u-\frac{1}{m}\right)-X_{n}\left(u-\frac{1}{m}\right)\right|^{2}\right) d u \\
& +10\left[T+\alpha^{2} \frac{T^{2 \alpha-1}}{2 \alpha-1}\right] \int_{0}^{t} \mathcal{G}\left(u, \mathbb{E}\left|X_{n}\left(u-\frac{1}{m}\right)-X_{n}\left(u-\frac{1}{n}\right)\right|^{2}\right) d u .
\end{aligned}
$$

In terms of Part 2, we have

$$
\begin{aligned}
J_{1}+J_{4} \leq & 10\left[T+\alpha^{2} \frac{T^{2 \alpha-1}}{2 \alpha-1}\right] \int_{0}^{t} \mathcal{G}\left(s, \mathbb{E}\left(\sup _{0 \leq u \leq s}\left|X_{m}(u)-X_{n}(u)\right|^{2}\right)\right) d s \\
& +10\left[T+\alpha^{2} \frac{T^{2 \alpha-1}}{2 \alpha-1}\right] \\
& \times \int_{0}^{t} \mathcal{G}\left(s, C_{4}\left(\frac{1}{n}-\frac{1}{m}\right)+C_{6}\left(\frac{1}{n}-\frac{1}{m}\right)^{2 \alpha}+C_{5} \sum_{s-1 / n<t_{j}<s-1 / m} C\right) d s .
\end{aligned}
$$

Similarly to (10), by the B-D-G inequality, Lemma 2.1, and condition (H2) we have

$$
\begin{aligned}
J_{2}+J_{3} \leq & 20 \mathbb{E} \int_{0}^{t}\left|\sigma_{1}\left(u, X_{m}\left(u-\frac{1}{m}\right)\right)-\sigma_{1}\left(u, X_{n}\left(u-\frac{1}{n}\right)\right)\right|^{2} d u \\
& +10 H T^{2 H-1} \mathbb{E} \int_{0}^{t}\left|g\left(u, X_{m}\left(u-\frac{1}{m}\right)\right)-g\left(u, X_{n}\left(u-\frac{1}{n}\right)\right)\right|^{2} d u \\
& +20 T \mathbb{E} \int_{0}^{t}\left|D_{u}^{\psi}\left(g\left(u, X_{m}\left(u-\frac{1}{m}\right)\right)-g\left(u, X_{n}\left(u-\frac{1}{n}\right)\right)\right)\right|^{2} d u
\end{aligned}
$$




$$
\begin{aligned}
& \leq 40 \mathbb{E} \int_{0}^{t}\left|\sigma_{1}\left(u, X_{m}\left(u-\frac{1}{m}\right)\right)-\sigma_{1}\left(u, X_{n}\left(u-\frac{1}{m}\right)\right)\right|^{2} d u \\
& +40 \mathbb{E} \int_{0}^{t}\left|\sigma_{1}\left(u, X_{n}\left(u-\frac{1}{m}\right)\right)-\sigma_{1}\left(u, X_{n}\left(u-\frac{1}{n}\right)\right)\right|^{2} d u \\
& +20 H T^{2 H-1} \mathbb{E} \int_{0}^{t}\left|g\left(u, X_{m}\left(u-\frac{1}{m}\right)\right)-g\left(u, X_{n}\left(u-\frac{1}{m}\right)\right)\right|^{2} d u \\
& +20 H T^{2 H-1} \mathbb{E} \int_{0}^{t}\left|g\left(u, X_{n}\left(u-\frac{1}{m}\right)\right)-g\left(u, X_{n}\left(u-\frac{1}{n}\right)\right)\right|^{2} d u \\
& +40 T \mathbb{E} \int_{0}^{t}\left|D_{u}^{\psi}\left(g\left(u, X_{m}\left(u-\frac{1}{m}\right)\right)-g\left(u, X_{n}\left(u-\frac{1}{m}\right)\right)\right)\right|^{2} d u \\
& +40 T \mathbb{E} \int_{0}^{t}\left|D_{u}^{\psi}\left(g\left(u, X_{n}\left(u-\frac{1}{m}\right)\right)-g\left(u, X_{n}\left(u-\frac{1}{n}\right)\right)\right)\right|^{2} d u \\
& \leq 20\left[2+2 T+H T^{2 H-1}\right] \int_{0}^{t} \mathcal{G}\left(u, \mathbb{E}\left|X_{m}\left(u-\frac{1}{m}\right)-X_{n}\left(u-\frac{1}{m}\right)\right|^{2}\right) d u \\
& +20\left[2+2 T+H T^{2 H-1}\right] \int_{0}^{t} \mathcal{G}\left(u, \mathbb{E}\left|X_{n}\left(u-\frac{1}{m}\right)-X_{n}\left(u-\frac{1}{n}\right)\right|^{2}\right) d u \\
& \leq 20\left[2+2 T+H T^{2 H-1}\right] \int_{0}^{t} \mathcal{G}\left(s, \mathbb{E}\left(\sup _{0 \leq u \leq s}\left|X_{m}(u)-X_{n}(u)\right|^{2}\right)\right) d s \\
& +20\left[2+2 T+H T^{2 H-1}\right] \\
& \times \int_{0}^{t} \mathcal{G}\left(s, C_{4}\left(\frac{1}{n}-\frac{1}{m}\right)+C_{6}\left(\frac{1}{n}-\frac{1}{m}\right)^{2 \alpha}+C_{5} \sum_{s-1 / n<t_{j}<s-1 / m} C\right) d s .
\end{aligned}
$$

Finally, for $J_{5}$, by condition (H3) we obtain

$$
\begin{aligned}
& J_{5} \leq 5 m \sum_{j=1}^{m} \mathbb{E}\left|I_{j}\left(X_{m}\left(u_{j}-\frac{1}{m}\right)\right)-I_{j}\left(X_{n}\left(u_{j}-\frac{1}{n}\right)\right)\right|^{2} \\
& \leq 10 m \sum_{j=1}^{m} \mathbb{E}\left|I_{j}\left(X_{m}\left(u_{j}-\frac{1}{m}\right)\right)-I_{j}\left(X_{n}\left(u_{j}-\frac{1}{m}\right)\right)\right|^{2} \\
& +10 m \sum_{j=1}^{m} \mathbb{E}\left|I_{j}\left(X_{n}\left(u_{j}-\frac{1}{m}\right)\right)-I_{j}\left(X_{n}\left(u_{j}-\frac{1}{n}\right)\right)\right|^{2} \\
& \leq 10 m \sum_{j=1}^{m}\left(d_{j}\right)^{2} \mathbb{E}\left|X_{m}\left(u_{j}-\frac{1}{m}\right)-X_{n}\left(u_{j}-\frac{1}{m}\right)\right|^{2} \\
& +10 m \sum_{j=1}^{m}\left(d_{j}\right)^{2} \mathbb{E}\left|X_{n}\left(u_{j}-\frac{1}{m}\right)-X_{n}\left(u_{j}-\frac{1}{n}\right)\right|^{2} \\
& \leq 10 m \sum_{j=1}^{m}\left(d_{j}\right)^{2}\left(C_{4}\left(\frac{1}{n}-\frac{1}{m}\right)+C_{6}\left(\frac{1}{n}-\frac{1}{m}\right)^{2 \alpha}+C_{5} \sum_{s-1 / n<t_{j}<s-1 / m} C\right) \\
& +10 m \sum_{j=1}^{m}\left(d_{j}\right)^{2} \mathbb{E}\left(\sup _{0 \leq u \leq t}\left|X_{m}(u)-X_{n}(u)\right|^{2}\right) .
\end{aligned}
$$


Combining Eqs. (9)-(12), we conclude

$$
\begin{aligned}
\mathbb{E}\left(\sup _{0 \leq s \leq t}\left|X_{m}(s)-X_{n}(s)\right|^{2}\right) \leq & C_{7} \int_{0}^{t} \mathcal{G}\left(s, \mathbb{E}\left(\sup _{0 \leq u \leq s}\left|X_{m}(u)-X_{n}(u)\right|^{2}\right)\right) d s \\
& +C_{7} \int_{0}^{t} \mathcal{G}\left(s, C_{4}\left(\frac{1}{n}-\frac{1}{m}\right)+C_{6}\left(\frac{1}{n}-\frac{1}{m}\right)^{2 \alpha}\right. \\
& \left.+C_{5} \sum_{s-1 / n<t_{j}<s-1 / m} C\right) d s \\
& +C_{8}\left(C_{4}\left(\frac{1}{n}-\frac{1}{m}\right)+C_{6}\left(\frac{1}{n}-\frac{1}{m}\right)^{2 \alpha}\right. \\
& \left.+C_{5} \sum_{s-1 / n<t_{j}<s-1 / m} C\right)
\end{aligned}
$$

where $C_{7}=\frac{10 C_{1}}{1-10 m \sum_{j=1}^{m}\left(d_{j}\right)^{2}}$ and $C_{8}=\frac{10 m \sum_{j=1}^{m}\left(d_{j}\right)^{2}}{1-10 m \sum_{j=1}^{m}\left(d_{j}\right)^{2}}$ are positive constants. Let

$$
\mathcal{M}(t)=\lim _{m, n \rightarrow \infty} \mathbb{E}\left(\sup _{0 \leq s \leq t}\left|X_{m}(s)-X_{n}(s)\right|^{2}\right)
$$

Then Eqs. (13) and (14), together with Fatou's lemma, yield

$$
\begin{aligned}
\mathcal{M}(t) \leq & C_{7} \lim _{m, n \rightarrow \infty} \int_{0}^{t} \mathcal{G}\left(s, C_{4}\left(\frac{1}{n}-\frac{1}{m}\right)+C_{6}\left(\frac{1}{n}-\frac{1}{m}\right)^{2 \alpha}+C_{5} \sum_{s-1 / n<t_{j}<s-1 / m} C\right) d s \\
& +C_{8} \lim _{m, n \rightarrow \infty}\left(C_{4}\left(\frac{1}{n}-\frac{1}{m}\right)+C_{6}\left(\frac{1}{n}-\frac{1}{m}\right)^{2 \alpha}+C_{5} \sum_{s-1 / n<t_{j}<s-1 / m} C\right) \\
& +C_{7} \lim _{m, n \rightarrow \infty} \int_{0}^{t} \mathcal{G}\left(s, \mathbb{E}\left(\sup _{0 \leq u \leq s}\left|X_{m}(u)-X_{n}(u)\right|^{2}\right)\right) d s \\
\leq & C_{7} \int_{0}^{t} \mathcal{G}\left(s, \lim _{m, n \rightarrow \infty} \mathbb{E}\left(\sup _{0 \leq u \leq s}\left|X_{m}(u)-X_{n}(u)\right|^{2}\right)\right) d s \\
\leq & C_{7} \int_{0}^{t} \mathcal{G}(s, \mathcal{M}(s)) d s,
\end{aligned}
$$

where we have used the facts that $\mathcal{G}(s, 0)=0$ and $\sum_{s-1 / n<t_{j}<s-1 / m} C \rightarrow 0$ as $n, m \rightarrow \infty$. Lastly, through Eq. (15) and condition (H1), we immediately get

$$
\mathcal{M}(t)=\lim _{m, n \rightarrow \infty} \mathbb{E}\left(\sup _{0 \leq s \leq t}\left|X_{m}(s)-X_{n}(s)\right|^{2}\right)=0,
$$

indicating that $\left\{X_{n}(t)\right\}_{n \geq 1}$ is a Cauchy sequence. The Borel-Cantelli lemma shows that, as $n \rightarrow \infty, X_{n}(t) \rightarrow X(t)$ uniformly for $t \in[0, T]$. Hence taking limits on both sides of Eq. (3), we obtain that $X(t), t \in[0, T]$, is a solution to Eq. (1) with the property $\mathbb{E}\left(\sup _{0 \leq s \leq t}|X(s)|^{2}\right)<$ $\infty$ for all $t \in[0, T]$, and this completes the proof of the existence. Now the uniqueness of solution can be obtained by the same procedure as Part 3 . Therefore the proof of Theorem 3.1 is completed. 


$$
\begin{aligned}
& \text { If } g \equiv 0 \text {, then system (1) becomes } \\
& \qquad \begin{array}{r}
d X(t)=b(t, X(t)) d t+\sigma_{1}(t, X(t)) d W(t) \\
\quad+\sigma_{2}(t, X(t))(d t)^{\alpha}, \quad t \in[0, T], t \neq t_{j}, \alpha \in(0,1), \\
\triangle X\left(t_{j}\right)=X\left(t_{j}^{+}\right)-X\left(t_{j}^{-}\right)=I_{j}\left(X\left(t_{j}\right)\right), \quad j=1,2, \ldots, m, \\
X(0)=X_{0} \in \mathbb{R}^{d} .
\end{array}
\end{aligned}
$$

Corollary 3.1 Let hypotheses (H1)-(H3) be satisfied, and let $X_{0}$ be independent of the Wiener process $W(s), s>0$, with finite second moment. Then there exists a unique solution $X(t)$ to Eq. (16), provided that $8 m \sum_{j=1}^{m}\left(d_{j}\right)^{2}<1$.

Remark 3.1 If $I_{j}(\cdot) \equiv 0(j=1,2, \ldots, m)$ in Eq. (16), then Corollary 3.1 is consistent with Theorem 3.1 in Abouagwa and Li [1]. Therefore Corollary 3.1 extends and improves some results in [1].

$$
\begin{aligned}
& \text { If } \sigma_{1}=\sigma_{2} \equiv 0 \text {, then Eq. (1) reduces to } \\
& \qquad \begin{array}{l}
d X(t)=b(t, X(t)) d t+g(t, X(t)) d W^{H}(t), \quad t \in[0, T], t \neq t_{j}, \\
\triangle X\left(t_{j}\right)=X\left(t_{j}^{+}\right)-X\left(t_{j}^{-}\right)=I_{j}\left(X\left(t_{j}\right)\right), \quad j=1,2, \ldots, m, \\
X(0)=X_{0} \in \mathbb{R}^{d} .
\end{array}
\end{aligned}
$$

Corollary 3.2 Let hypotheses (H1)-(H3) be satisfied, and let $X_{0}$ be independent of the fBm $W^{H}(s)(s>0, H>1 / 2)$ with finite second moment. Then there exists a unique solution $X(t)$ to Eq. (17), provided that $6 m \sum_{j=1}^{m}\left(d_{j}\right)^{2}<1$.

Remark 3.2 It should be mentioned that Xue et al. [36] established the existence and uniqueness results to Eq. (17) without impulses $\left(I_{j}(\cdot)=0(j \equiv 1,2, \ldots, m)\right)$ under conditions (H1) and (H2) by means of successive approximation. Our results are obtained for Eq. (17) with impulses by means of Carathéodory approximation. Hence Corollary 3.2 is an extension and improvement of Theorem A in [36].

Remark 3.3 Replacing $\mathcal{G}(t, v)$ in hypothesis $(\mathrm{H} 1)$ by $\mathcal{G}(t, v)=\lambda(t) \overline{\mathcal{G}}(v), t \in[0, T]$, where $\lambda(t) \geq 0$ is locally integrable, and $\overline{\mathcal{G}}(v):[0, \infty) \longrightarrow[0, \infty)$ is a concave nondecreasing function with $\overline{\mathcal{G}}(0)=0, \overline{\mathcal{G}}(v)>0$ for $v>0$, and $\int_{0+} \frac{1}{\overline{\mathcal{G}}(v)} d v=+\infty$. Then Corollaries 3.1 and 3.2 extend and improve some results in Abouagwa et al. [4] and Pei and Xu [28] $(\lambda(t)=1)$, respectively.

\section{An application}

In this section, as an application of the obtained results, we provide the following impulsive stochastic fractional Burgers differential equations with Dirichlet boundary condi- 
tions driven by a standard Brownian motion and independent $\mathrm{fBm}$ :

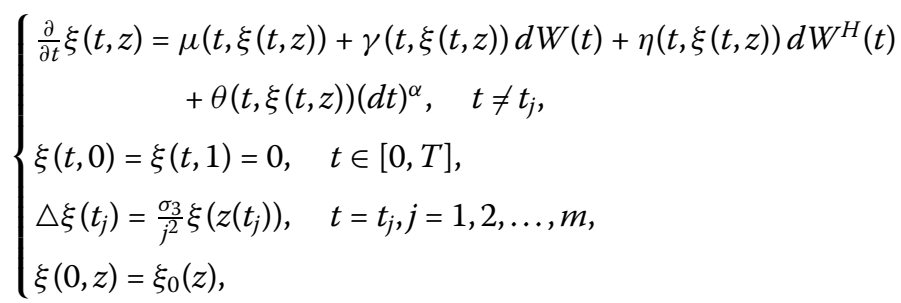

where $0 \leq z \leq 1,0<\alpha<1, \xi_{0}(z) \in \mathbb{R}^{d}, W(t)$ and $W^{H}(t)$ are two independent $m$ dimensional Brownian motion and $\mathrm{fBm}$, respectively, $\mu, \theta:[0, T] \times \mathbb{R}^{d} \longrightarrow \mathbb{R}^{d}$ and $\gamma, \eta$ : $[0, T] \times \mathbb{R}^{d} \longrightarrow \mathbb{R}^{d \times m}$ are continuous functions, and $\sigma_{3}>0$.

Let $X(t)(z)=\xi(t, z)$ and

$$
\begin{aligned}
& b(t, X(t))(z)=\mu(t, \xi(t, z)), \\
& \sigma_{1}(t, X(t))(z)=\gamma(t, \xi(t, z)), \\
& g(t, X(t))(z)=\eta(t, \xi(t, z)), \\
& \sigma_{2}(t, X(t))(z)=\theta(t, \xi(t, z)), \\
& I_{j}\left(X\left(t_{j}\right)\right)=\frac{\sigma_{3}}{j^{2}} \xi\left(z\left(t_{j}\right)\right) .
\end{aligned}
$$

Then problem (1) is an abstract version of problem (18). We can choose suitable functions $\mu, \gamma, \eta, \theta$ such that conditions (H1)-(H3) are satisfied. Then by Theorem 3.1 problem (18) has a unique solution.

\section{Acknowledgements}

The authors are very grateful to the editor and anonymous referees for their valuable comments and suggestions, which have led to an improved presentation of the paper.

\section{Funding}

This work was supported by National Natural Science Foundation of China (No. 11771161).

\section{Availability of data and materials}

The paper did not produce any datasets.

\section{Competing interests}

There are no competing interests among the authors.

Authors' contributions

In this paper, all authors contributed equally. The authors read and approved the submission of the paper.

\section{Publisher's Note}

Springer Nature remains neutral with regard to jurisdictional claims in published maps and institutional affiliations.

Received: 30 July 2019 Accepted: 27 January 2020 Published online: 04 February 2020

\section{References}

1. Abouagwa, M., Li, J:: Approximation properties of solutions to Itô-Doob stochastic fractional differential equations with non-Lipschitz coefficients. Stoch. Dyn. 19, 1950029 (2019)

2. Abouagwa, M., Li, J.: Stochastic fractional differential equations driven by Lévy noise under Carathéodory conditions. J. Math. Phys. 60, 022701 (2019)

3. Abouagwa, M., Li, J.: G-Neutral stochastic differential equations with variable delay and non-Lipschitz coefficients. Discrete Contin. Dyn. Syst., Ser. B 25, 1583-1606 (2020)

4. Abouagwa, M., Liu, J., Li, J.: Carathéodory approximations and stability of solutions to non-Lipschitz stochastic fractional differential equations of Itô-Doob type. Appl. Math. Comput. 329, 143-153 (2018) 
5. Agarwal, P., Deniz, S., Jain, S., Alderremy, A.A., Aly, S.: A new analysis of a partial differential equation arising in biology and population genetics via semi analytical techniques. Phys. A, Stat. Mech. Appl. (2019).

https://doi.org/10.1016/j.physa.2019.122769

6. Ahmed, H.M.: Sobolev-type fractional stochastic integrodifferential equations with nonlocal conditions in Hilbert space. J. Theor. Probab. 30, 771-783 (2017)

7. Alos, E., Nualart, D.: Stochastic calculus with respect to the fractional Brownian motion. Ann. Probab. 29, 766-801 (2001)

8. Benchaabane, A., Sakthivel, R.: Sobolev-type fractional stochastic differential equations with non-Lipschitz coefficients. J. Comput. Appl. Math. 312, 65-73 (2017)

9. Biagini, F., Hu, Y., Øksendal, B., Zhang, T.: Stochastic Calulus for Fractional Brownian Motion and Applications. Springer, London (2008)

10. Boudaoui, A., Caraballo, T., Ouahab, A.: Existence of mild solutions to stochastic delay evolution equations with a fractional Brownian motion and impulses. Stoch. Anal. Appl. 33, 244-258 (2015)

11. Boudaoui, A., Caraballo, T., Ouahab, A.: Impulsive neutral functional differential equations driven by a fractional Brownian motion with unbounded delay. Appl. Anal. 95, 2039-2062 (2016)

12. Cui, J., Yan, L.: Existence result for fractional neutral stochastic integro-differential equations with infinite delay. J. Phys. A, Math. Theor. 44, 335201 (2011)

13. Deng, S., Shu, X.-B., Mao, J.: Existence and exponential stability for impulsive neutral stochastic functional differential equations driven by fBm with noncompact semigroup via Mönch fixed point. J. Math. Anal. Appl. 467, 398-420 (2018)

14. Dung, N.T.: Neutral stochastic differential equations driven by fractional Brownian motion with impulsive effects and varying-time delays. J. Korean Stat. Soc. 43, 599-608 (2014)

15. Hammouch, Z., Mekkaoui, T., Agarwal, P.: Optical solitons for the Calogero-Bogoyavlenskii-Schiff equation in $(2+1)$ dimensions with time-fractional conformable derivative. Eur. Phys. J. Plus 133, 248 (2018)

16. Hu, Y., Øksendal, B.: Fractional white noise calculus and application to finance. Infin. Dimens. Anal. Quantum Probab. Relat. Top. 6, 1-32 (2003)

17. Jumarie, G.: On the representation of fractional Brownian motion as an integral with respect to $(d t)^{a}$. Appl. Math. Lett. $18,739-748(2005)$

18. Kalamani, P., Baleanu, D., Selvarasu, S., Arjunan, M.M.: On existence results for impulsive fractional neutral stochastic integro-differential equations with nonlocal and state-dependent delay conditions. Adv. Differ. Equ. 2016, 163 (2016)

19. Kerboua, M., Debbouche, A., Baleanu, D.: Approximate controllability of Sobolev-type nonlocal fractional stochastic dynamic systems in Hilbert spaces. Abstr. Appl. Anal. 2013, Article ID 262191 (2013)

20. Kerboua, M., Fateh, E., Baleanu, D.: Stochastic fractional perturbed control systems with fractional Brownian motion and Sobolev stochastic nonlocal conditions. Collect. Math. 69, 283-296 (2018)

21. Kolmogorov, A.N.: The Wiener spiral and some other interesting curves in Hilbert space. Dokl. Akad. Nauk SSSR 26 115-118 (1940)

22. Lakshmikantham, V., Bainov, D.D., Simeonov, P.S.: Theory of Impulsive Differential Equations. World Scientific, Singapore (1989)

23. Li, K.X.: Stochastic delay fractional evolution equations driven by fractional Brownian motion. Math. Methods Appl. Sci. 38, 1582-1591 (2015)

24. Liu, J.F., Yan, L.T., Cang, Y.Q.: On a jump-type stochastic fractional partial differential equation with fractional noises. Nonlinear Anal. 75, 6060-6070 (2012)

25. Mishura, Y.S.: Stochastic Calculus for Fractional Brownian Motion and Related Processes. Springer, Berlin (2008)

26. Morales-Delgado, V.F., Gómez-Aguilar, J.F., Saad, K.M., Khan, M.A., Agarwal, P.: Analytic solution for oxygen diffusion from capillary to tissues involving external force effects: a fractional calculus approach. Phys. A, Stat. Mech. Appl. 523, 48-65 (2019)

27. Pedjeu, G.C., Ladde, G.S.: Stochastic fractional differential equations: modeling, method and analysis. Chaos Solitons Fractals 34, 279-293 (2012)

28. Pei, B., Xu, Y.: On the non-Lipschitz stochastic differntial equations driven by fractional Brownian motion. Adv. Differ. Equ. 2016, 194 (2016)

29. Rekhviashvili, S., Pskhu, A., Agarwal, P., Jain, S.: Application of the fractional oscillator model to describe damped vibrations. Turk. J. Phys. 43, 236-242 (2019)

30. Russo, F., Vallois, P.: Forward, backward and symmetric stochastic integration. Probab. Theory Relat. Fields 97, 403-421 (1993)

31. Sakthivel, R., Revathi, P., Ren, Y.: Existence of solutions for nonlinear fractional stochastic differential equations. Nonlinear Anal. 81, 70-86 (2013)

32. Salahshour, S., Ahmadian, A., Senu, N., Baleanu, D., Agarwal, P.: On analytical solutions of fractional differential equations with uncertainty: application to the Basset problem. Entropy 17, 885-902 (2015)

33. Shiryaev, A.N.: Essentials of Stochastic Finance: Facts, Models and Theory. World Scientific, Singapore (1999)

34. Taniguchi, T.: Successive approximations to solutions of stochastic differential equations. J. Differ. Equ. 96, 152-169 (1992)

35. Xu, L., Li, Z.: Stochastic fractional evolution equations with fractional Brownian motion and infinite delay. Appl. Math. Comput. 336, 36-46 (2018)

36. Xu, Y., Pei, B., Wu, J.L.: Stochastic averaging principle for different equations with non-Lipschitz coefficients driven by fractional Brownian motion. Stoch. Dyn. 17, 1720013 (2017) 